\title{
Negative-Tension Instability of Scroll Waves and Winfree Turbulence in the Oregonator Model
}

\author{
S. Alonso,* F. Sagués, and A. S. Mikhailov \\ Abteilung Physikalische Chemie, Fritz-Haber-Institut der Max-Planck-Gesellschaft, Faradayweg 4-6, \\ 14195 Berlin, Germany
}

Received: July 3, 2006; In Final Form: September 1, 2006

\begin{abstract}
Excitable media support self-organized scroll waves which can be unstable and give rise to three-dimensional wave chaos. Winfree turbulence of scroll waves results from the negative-tension instability of scroll waves; it plays an important role in the cardiac tissue where it may lead to ventricular fibrillation. By numerical simulations of the Oregonator model, we show that this instability and, thus, the Winfree turbulence may also be observed in the Belousov-Zhabotinsky reaction. The region in the parameter space, where the instability takes place, is determined, and a relationship between the negative-tension instability and the meandering behavior of spiral waves is found. The application of global periodic forcing to control such turbulence in the Oregonator model is discussed.
\end{abstract}

\section{Introduction}

The autocatalytic Belousov-Zhabotinsky reaction provides characteristic examples of wave behavior in excitable media. Experiments with the Belousov-Zhabotinsky reaction in thin layers exhibit such generic two-dimensional (2D) wave patterns as pacemakers ${ }^{1}$ or spiral waves; ${ }^{2}$ the transition to chemical turbulence due to spiral breakup has also been found. ${ }^{3}$ For the reaction in thick layers, three-dimensional (3D) effects become important, and patterns of scroll waves are observed ${ }^{4}$ which can be unstable and give rise to chaotic dynamics. ${ }^{5,6}$ Generally, the behavior of chemical waves in the Belousov-Zhabotinsky reaction is well described by the Oregonator model of this reaction. ${ }^{7}$ Similar kinds of patterns exist in a completely different excitable medium, i.e., in the cardiac tissue. ${ }^{8}$ There, electrical excitations propagate through the tissue as traveling waves which coordinate physiological contractions of the cardiac muscle, while spiral and scroll waves are related to pathological conditions. ${ }^{9}$ The heart is three-dimensional, and this is crucial for several types of arrhythmias. ${ }^{10}$ The close correspondence between the phenomena in cardiac tissue and in chemical excitable media provides an additional motivation for the studies of wave patterns in the Belousov-Zhabotinsky reaction. It is known that only thick preparations of cardiac tissue may show fibrillation, and, therefore, 3D effects must be important in this system. ${ }^{10}$ Having this in mind, it is interesting to look for the instabilities which only appear in 3D excitable media, when wave propagation in thin layers is completely stable. One of them is the negative tension instability associated with the expanding scroll rings. ${ }^{11,12}$ This instability was studied for a general Barkley model of excitable media in our previous publications. ${ }^{13,14}$ The negative-tension instability and the associated Winfree turbulence of scroll waves have not yet been observed in the experiments with the Belousov-Zhabotinsky reaction. Numerical simulations of scroll waves in the Oregonator model of the Belousov-Zhabotinsky reaction, based on the mechanism proposed by R. J. Field, E. Koros, and R. M. Noyes, ${ }^{15}$ show that such instability should also be possible in this classical system and determine the conditions for its experimental observation.
Scroll waves rotate around a nonexcited linear filament. This filament could be straight or curved and can be closed into a ring. Scroll rings found in the experiments are not stationary: they shrink and eventually disappear. ${ }^{16}$ However, analytical and numerical studies predict that scroll rings should not always shrink: they may also expand under weakly excitable conditions. ${ }^{17}$ The expansion of scroll rings means that small local perturbations of a linear filament, representing small curved pieces, grow, and thus the filaments are unstable, tending to stretch spontaneously. This effect is known as the negativetension instability. Such instability is purely $3 \mathrm{D}$, and, in the same $2 \mathrm{D}$ medium, rotating spiral waves may remain stable. The first numerical investigations of the expansion of scroll rings ${ }^{11}$ and the first studies of the negative-tension instability ${ }^{12}$ as well as the recent detailed explorations of the final turbulent state $^{13,14,18}$ were performed using abstract models of excitable media. Our study extends these investigations to the realistic Oregonator model of the Belousov-Zhabotinsky reaction.

Wave patterns in the Belousov-Zhabotinsky reaction can be easily controlled if a photosensitive modification of this reaction is chosen (see, e.g., ref 19). External optical forcing ${ }^{20}$ and feedback ${ }^{21}$ have been used to steer propagation of $2 \mathrm{D}$ waves in this system. Furthermore, several methods have been employed for the control of scroll waves. It was experimentally shown that gradients of temperature or illumination can affect the orientation $^{22}$ and the dynamics ${ }^{23}$ of scroll rings. Previous theoretical studies have further predicted that the negative tension instability can be controlled by application of periodic external forcing. ${ }^{13,24}$ Here, we extend these predictions by numerical simulations of the photosensitive Oregonator model under global periodic modulation of the illumination intensity.

In the next section the model and the employed numerical methods are described. Simulation results are reported in section 3 and are discussed in section 4 .

\section{The Oregonator Model}

In the last two decades, the Oregonator model has been widely used to predict, compare, and verify observations in the 

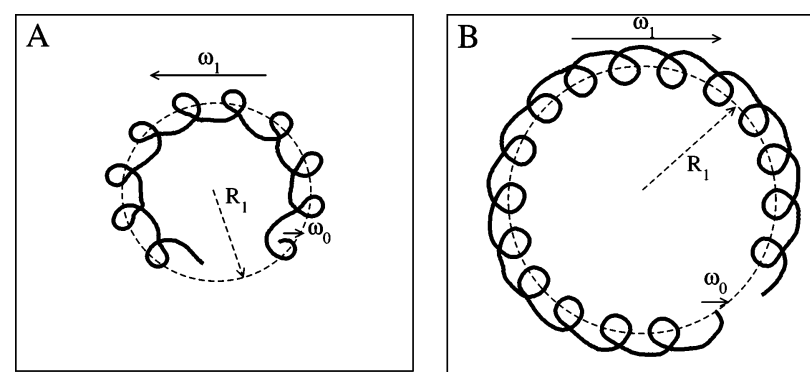

Figure 1. Motion of the tip of a meandering spiral wave with outward loops (A) and with inward loops (B). The solid arrows show the direction of motion around the loops $\left(\omega_{0}\right)$ and around the secondary circle $\left(\omega_{1}\right)$. Simulations are performed with the Oregonator model with $D_{u}=1, D_{v}=0.0, \epsilon=0.05, q=0.002, \phi=0$ and (A) $f=3.8$ or (B) $f=4.0$.

Belousov-Zhabotinsky reaction. 3D simulations with this model can help to guide experiments aimed at the observation of the negative-tension instability. Our numerical simulations are performed using the two-variables variant of the Oregonator model, ${ }^{7}$ modified to take into account illumination in the photosensitive version. ${ }^{25}$ The reaction-diffusion equations of this model are

$$
\begin{gathered}
\frac{\partial u}{\partial t}=D_{u} \nabla^{2} u+\frac{1}{\epsilon}\left(u(1-u)-\left((f v-\phi) \frac{u-q}{u+q}\right)\right) \\
\frac{\partial v}{\partial t}=D_{v} \nabla^{2} v+u-v
\end{gathered}
$$

where $u$ is the fast activator variable, and $v$ is the slow inhibitor variable. The variable $u$ corresponds to the local chemical concentration of $\mathrm{HBrO}_{2}$, and $v$ corresponds to the concentration of the catalyst of the reaction, i.e., ferroin for the classical version of the reaction and ruthenium bypyridyl in its photosensitive modification. The small parameter $\epsilon \ll 1$ represents the ratio of time scales of the dynamics of the fast and slow variables; $f$ is the stoichiometric parameter which is positive and typically smaller than $4,{ }^{26}$ and $q$ is another chemical parameter. $D_{u}$ and $D_{v}$ are, respectively, the dimensionless diffusion coefficients of $\mathrm{HBrO}_{2}$ and the catalyst. Choosing appropriate length units, we fix $D_{u}=1$. For the diffusion coefficient of the catalyst, we take the values $D_{v}=0.6$ in the aqueous solution, following ref 26 , and $D_{v}=0$ when the reaction takes place in a gel which immobilizes the catalyst and prevents its diffusion. ${ }^{27}$ The parameter $\phi$ is proportional to the intensity of the illumination.

Spiral waves in the Oregonator model were systematically studied for $D_{v}=0.6$ and $\phi=0$ by W. Jahnke and A. T. Winfree in $1991 .^{26}$ They determined the parameter regions of rigidly rotating, meandering, and chaotic spiral waves and described dynamical properties of spiral waves in these different regimes. Their main focus was on the meandering spiral waves. The tip of a meandering spiral wave performs composite motion with several frequencies, giving rise to flower patterns. In the simplest case, the tip follows a cycloidal trajectory representing a superposition of two circular motions with different frequencies. The main motion is a periodic rotation of the spiral tip around the unexcited region of the core with frequency $\omega_{0}$ and radius $R_{0}$ and corresponds to the small loops of both panels in Figure 1. Furthermore, the center of rotation of the spiral migrates around a circle of radius $R_{1}$, plotted by dashed lines in Figure 1. When the center of the spiral rotates around the second radius in the direction opposite to the motion of the tip around the circular core, the spiral tip meanders with outward loops (Figure
1,A). On the other hand, when the center of rotation and the tip of the spiral rotate in the same direction, the loops of the meander are inward oriented (Figure 1,B). Both regimes are observed in the Oregonator model. ${ }^{26,28}$ Equations 1 are dimensionless and are integrated using the finite-difference method with the temporal step $\Delta t=0.0015$ and the spatial step $\Delta x=$ 0.5 in all simulations. A grid of $256 \times 256$ points is used to integrate the equations in 2D. The linear size of the medium in our system is at least five times larger than the distance between two subsequent coils of the spiral waves, preventing the influence of boundaries on the spiral wave motion. No-flux boundary conditions are employed in all our simulations. For large meandering of spiral waves, the tip of the spiral would reach the boundary of the medium. To avoid such interactions with the boundaries, special moving boundaries conditions are then used to keep the tip of the spiral wave inside the square of $128 \times 128$ pixels. These moving boundaries conditions consist of adding a slice of the grid in the direction of the motion and removing at the same time a slice on the other side of the grid when the spiral waves leaves the square, thus keeping the tip inside the grid and far away from the boundaries (see ref 14). Typically, the waves just follow the spiral tip, and small modifications far away from the center do not affect the dynamics. In 2D media, the position of the tip of the spiral wave is detected using the condition of the minimal value of the crossgradient of activator and inhibitor fields, as in ref 28.

Scroll rings are 3D waves with axial symmetry. Therefore, they are independent of the angle in the cylindrical coordinates with the vertical axis coinciding with the symmetry direction of the ring. To determine whether a scroll ring would expand or shrink for the given medium parameters, it is sufficient to integrate the dynamic equations of the effective $2 \mathrm{D}$ system

$$
\begin{gathered}
\frac{\partial u}{\partial t}=D_{u}\left(\frac{\partial^{2}}{\partial r^{2}}+\frac{1}{r} \frac{\partial}{\partial r}+\frac{\partial^{2}}{\partial z^{2}}\right) u+ \\
\frac{1}{\epsilon}\left(u(1-u)-\left((f v-\phi) \frac{u-q}{u+q}\right)\right) \\
\frac{\partial v}{\partial t}=D_{v}\left(\frac{\partial^{2}}{\partial r^{2}}+\frac{1}{r} \frac{\partial}{\partial r}+\frac{\partial^{2}}{\partial z^{2}}\right) v+u-v
\end{gathered}
$$

which are obtained by assuming axial symmetry. Note that such reduced simulations are valid only at the initial stage of the expansion of scroll rings, because the negative-tension instability of the filament eventually leads to its deformations and the breakdown of the axial symmetry.

3D simulations of scroll waves with an initially straight filament are performed for a grid with $150 \times 150 \times 200$ elements with the same discretization quantities as in the 2D systems and with the nonflux boundaries conditions. For the computation of the Laplacian, not only the first neighbors but also the second neighbors are taken into account, by the use of the 19-point finite-difference scheme. We employ the condition of crossover of the isosurfaces $u_{\mathrm{f}}=0.15$ and $F\left(u_{\mathrm{f}}\right)=0$ to detect the filament (see ref 29). Isosurfaces corresponding to $u_{\mathrm{s}}=0.25$ with the additional condition $v_{\mathrm{s}}<0.05$, to show only the frontal part of the wave, are plotted in the 3D snapshots in the last part of the paper. A modulation of the parameter $f$ along the vertical direction $f(z)=f_{0}+f_{\mathrm{m}} \cos (2 \pi z / \lambda)$ is introduced during a time lapse $\Delta t=0.75$ when the scroll wave is already formed, to perturb the initially straight filament and study its stability. The values employed in our simulations are $f_{\mathrm{m}}=0.2$ and $\lambda=8.0$. 


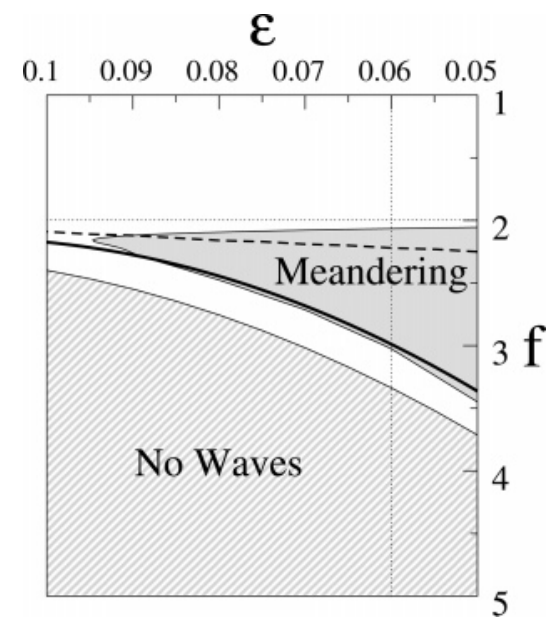

Figure 2. Phase diagram of the Oregonator model in the parameter plane $(f, \epsilon)$. The gray domain corresponds to meandering of spiral waves, the dashed light gray region to nonexcitable media, and the white area to rigidly rotating spiral waves. The medium is oscillatory above the dashed line and excitable below it. The bold line shows the boundary between the regions with positive and negative tension of the filament. For the meaning of the dotted lines, see Figure 6. Other parameters of the model are $D_{u}=1, D_{v}=0.6, q=0.002$, and $\phi=0$.

\section{Numerical Investigations}

The negative-tension instability, predicted in $1987,{ }^{17}$ was observed in numerical simulations of the FitzHugh-Nagumo model. ${ }^{12}$ Subsequently, the parameter region with negative tension was identified in the phase diagram of the Barkley model. This region lies between the region of meandering of 2D spiral waves and the nonexcitable domain. ${ }^{13}$ Below, we first determine the corresponding regions with the negative tension of filaments in the parameter space of the Oregonator model. After that, full 3D simulations are performed to observe the evolution of the filament and the final Winfree turbulence. At the end, we consider the effect of a periodic external forcing of scroll waves by periodically varying the model parameter that specifies the illumination intensity.

3.1. The Phase Diagrams. In the previous studies of the Oregonator model, its parameter regions with rigid rotation of spiral waves and meandering of spirals were determined. ${ }^{26}$ These results serve as the initial point in our current investigations. Figure 2 shows the phase diagram of the Oregonator model in the parameter plane of $f$ and $\epsilon$, keeping other parameters constant. Inside the region marked "no waves", wave propagation is not possible. To find the boundary of this region, we performed simulations of one-dimensional pulses. In the region of the parameter space where spiral waves were possible, two principal types of dynamics were observed, i.e., rigidly rotating and meandering spiral waves. Rigid spiral waves rotate around a circular core with frequency $\omega_{0}$. The size of the core depends on the excitability of the medium: weak excitability implies a large radius and long periods of rotation and high excitability implies a small radius and short periods of rotation. Meandering spiral waves have two or several frequencies involved in the motion of the tip. With only two frequencies $\omega_{1}$ and $\omega_{0}$, the tip motion is cycloidal as in Figure 1. The motion becomes more complex when more frequencies are involved in the meandering.

The phase diagram of the Oregonator model, shown in Figure 2 , is essentially the same as given in ref 26. To favor comparison, notice that $f$ and $\epsilon$ increase respectively from top to bottom and right to left. However, we include here two additional lines. The boundary separating parameter regions with oscillatory and excitable dynamics is indicated by the dashed

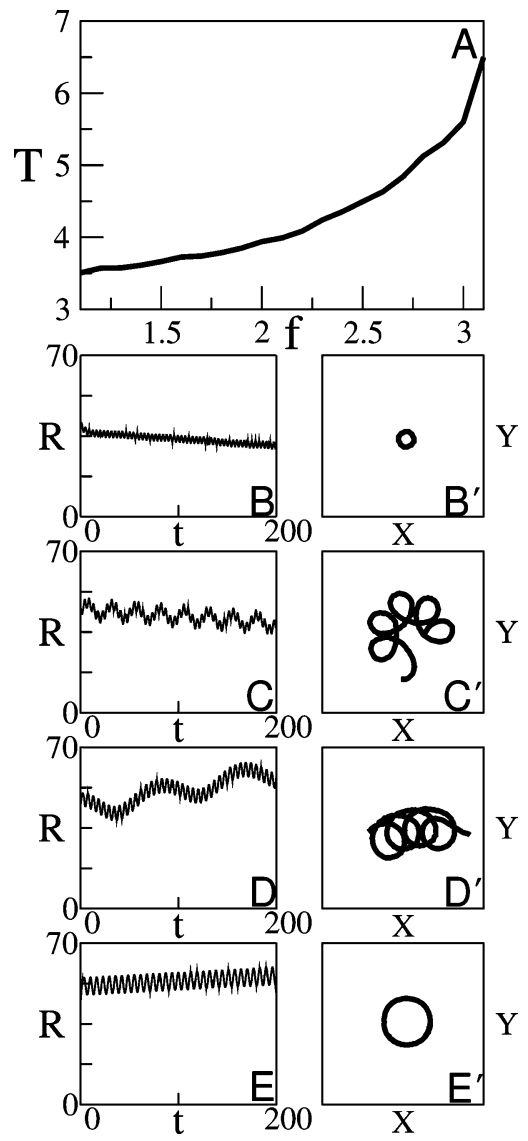

Figure 3. Dynamics of scroll rings and spiral waves for different values of the parameter $f$, with $\epsilon=0.06$ and other parameters as in Figure 2 . (A) The period of the spiral waves as a function of $f$. Examples of the evolution of the radius of scroll rings $(\mathrm{B}-\mathrm{E})$ and the dynamics of spiral waves $\left(\mathrm{B}^{\prime}-\mathrm{E}^{\prime}\right)$, for $\left(\mathrm{B}, \mathrm{B}^{\prime}\right) f=1.75,\left(\mathrm{C}, \mathrm{C}^{\prime}\right) f=2.5$, $\left(\mathrm{D}, \mathrm{D}^{\prime}\right) f=3.015$, and $\left(\mathrm{E}, \mathrm{E}^{\prime}\right) f=3.1$.

line (see ref 30). The bold line shows the boundary separating regions with expansion and collapse of scroll rings. Above this line in Figure 2, scroll rings shrink. ${ }^{16}$ Below this line, scroll rings grow, and the tension of the filament of the scroll waves is negative, leading to the chaotic regime known as Winfree turbulence.

The loops of the meandering motion can be oriented toward the center of the large radius (Figure 1,B) or outside (Figure $1, \mathrm{~A})$. Separating the regions of meandering with inward and outward loops, there is a boundary on which linear drift of spiral waves is observed. We have found in our numerical simulations that this boundary always coincides, inside the meandering region, with the boundary separating the regions with expansion and collapse of scroll rings. Thus, meandering with outward loops in the 2D media corresponds in three dimensions to the collapse of scroll rings, and meandering with inward loops corresponds to the expansion of scroll rings. Examples of the evolution of the radius of scroll rings for different values of the parameter $f$ are displayed in Figure 3. To help understanding these examples, we also show in Figure 3,A the dependence of the main period of rotation of spiral waves, associated with the frequency $\omega_{0}$ inside the loop of the meandering motion, on the parameter $f$. As expected, the period of spiral waves increases as $f$ increases, and the medium becomes less excitable. Under high excitability, the radius decreases with time and the scroll ring collapses (Figure 3,B). In the same 2D medium, spiral waves are rigidly rotating around a small core (Figure 3,B'). When the excitability is decreased and the parameter region with meandering of spiral waves is entered, the scroll ring 
$\varepsilon$

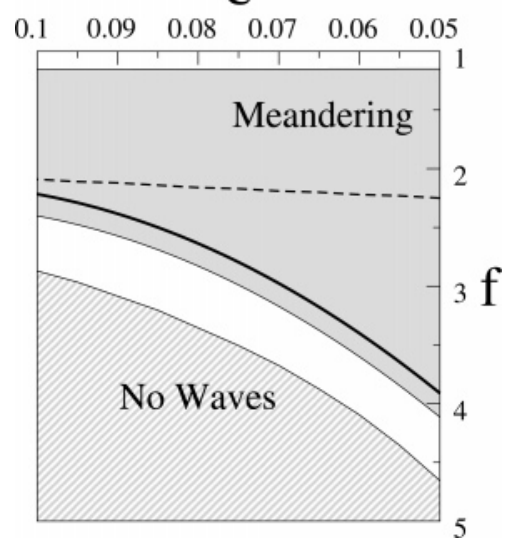

Figure 4. Phase diagram of the Oregonator model in the parameter plane $(f, \epsilon)$. The gray domain corresponds to meandering of spiral waves, the dashed light gray region to nonexcitable media, and the white area to rigidly rotating spiral waves. The medium is oscillatory above the dashed line and excitable below it. The bold line shows the boundary between the regions with positive and negative tension of the filament. Other parameters of the model are $D_{u}=1, D_{v}=0, q=$ 0.002 , and $\phi=0$.

continues to shrink, although its radius exhibits also some periodic time modulation (Figure 3,C). Now, the spiral wave tip performs a cycloidal motion with outward loops (Figure $\left.3, C^{\prime}\right)$. This cycloidal motion is superposed with the collapse of the ring, as seen in Figure 3,C. When the parameter $f$ is further increased, the meandering of the spiral wave becomes different and the loops are then looking inward (Figure 3,D'). Under these conditions, the scroll ring expands, while also showing some periodic modulation related to the meandering (Figure 3,D). Finally, when excitability of the medium is weak, the spiral wave again rotates rigidly around a core with a large radius and with a long period (Figure 3, $\mathrm{E}^{\prime}$ ). Now, the scroll ring steadily expands (Figure 3,E), and this expansion gives rise to turbulence. ${ }^{14}$ Numerical simulations in Figure 2 were done using a relatively large value of the inhibitor diffusion $\left(D_{v}=0.6\right)$, to compare these results with those of ref 26 . However, spiral and scroll waves can also be observed in the Belousov-Zhabotinsky reaction inside gels or porous medium. Gels typically prevent diffusion of the inhibitor. This effect can be important because we have shown previously ${ }^{14}$ that decrease of the diffusion coefficient of the inhibitor can induce the negative-tension instability of filaments. In the opposite limit $D_{u}=D_{v}$, scroll rings always collapse at rate $-D_{u} / R$ (see ref 31$)$.

Therefore, simulations have been repeated for the immobile inhibitor $\left(D_{v}=0\right)$. Their results are presented in Figure 4 . The meandering region occupies the main part of the phase diagram. It can be noticed that the size of the region corresponding to negative tension is increased now, and, importantly, the meandering region with inward loops is much larger under this condition. As before, the boundary between expansion and collapse of scroll rings coincides with the boundary between the regions with inward and outward loops inside the domain of meandering of spiral waves. The boundary between oscillatory and excitable dynamics remains the same because it does not depend on diffusion coefficients. Increasing the parameter $f$ lowers excitability of the medium, and spiral waves rotate slower, as seen in Figure 5,A. For high excitability, spiral waves rotate rigidly, but meandering soon develops. Meandering with different numbers of loops is observed, five loops in Figure $5, \mathrm{~B}^{\prime}$ or six in Figure 5, $\mathrm{C}^{\prime}$, but scroll rings shrink in both cases, see Figure 5,B,C. However, in agreement with Figure 3, when the loops of the meandering become inward (Figure 5,H), scroll

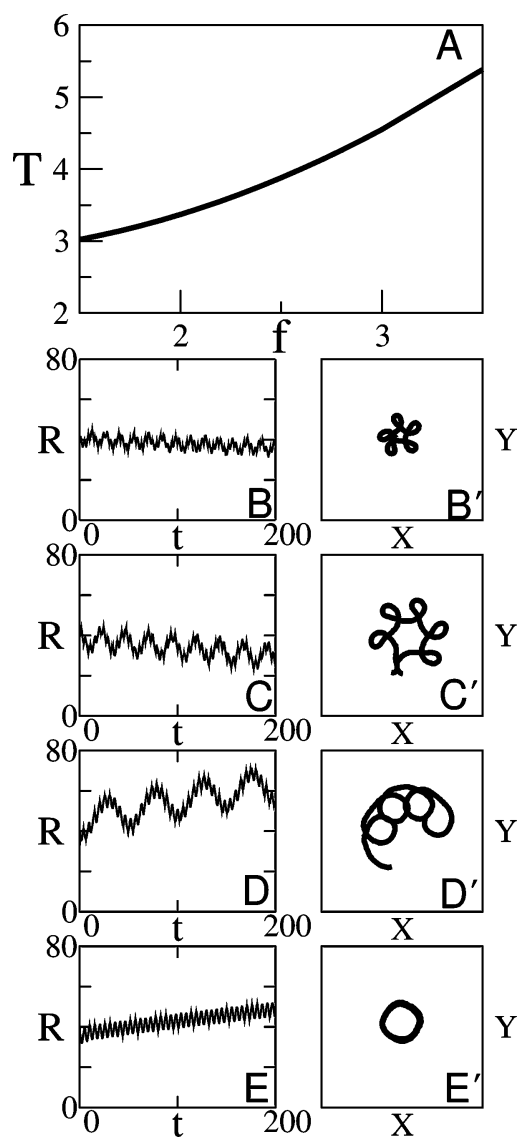

Figure 5. Dynamics of scroll rings and spiral waves for different values of the parameter $f$, with $\epsilon=0.05$ and other parameters as in Figure 4 . (A) The period of the spiral waves as a function of $f$. Examples of the evolution of the radius of scroll rings $(\mathrm{B}-\mathrm{E})$ and the dynamics of spiral waves $\left(\mathrm{B}^{\prime}-\mathrm{E}^{\prime}\right)$, for $\left(\mathrm{B}, \mathrm{B}^{\prime}\right) f=2.5,\left(\mathrm{C}, \mathrm{C}^{\prime}\right) f=3,\left(\mathrm{D}, \mathrm{D}^{\prime}\right) f=3.5$, and (E, $\left.\mathrm{E}^{\prime}\right) f=3.6$.

rings begin to expand (Figure 5,D). For still weaker excitabilities where no meandering is observed, scroll rings also expand (Figure 5,E).

The above numerical simulations have been performed using the original version of the two-variables Oregonator model without taking into account illumination (i.e., by formally putting $\phi=0$ in the considered model). The modified version of the Oregonator system describing the photosensitive BelousovZhabotinsky reaction includes parameter $\phi$, which is proportional to the illumination intensity. For higher values of this parameter, excitability of the medium decreases, and, starting from some critical value, wave propagation is no longer possible. The effect of the variation of the parameter $\phi$, as seen in Figure 6,A, is similar to that of the increase of the parameter $f$ (cf. Figure 2).

In Figure 6, phase diagrams of the Oregonator model in the parameter planes $(\epsilon, f)$ and $(\phi, f)$ are displayed. The limits of $\phi$ $\rightarrow 0$ in both figures correspond to the dotted lines in Figure 2 . For $f=2$ (Figure 6,A), spiral waves rotate rigidly in the oscillatory media close to the transition to meandering of spiral waves. The meandering region is reduced here to a small domain of the parameter plane, although it grows for the smaller values of $\epsilon$. Meandering with inward loops is no longer observed, and the transition from positive to negative tension occurs outside the meandering region. This is another indication of the relationship between meandering motion with inward loops in $2 \mathrm{D}$ and the negative-tension instability of filaments in 3D.

The phase diagram in Figure 6,B is plotted in the parameter plane $(\phi, f)$, while keeping $\epsilon=0.06$; it corresponds in the limit $\phi \rightarrow 0$ to the vertical dotted line in Figure 2. The diagram is 


\section{$\varepsilon$}

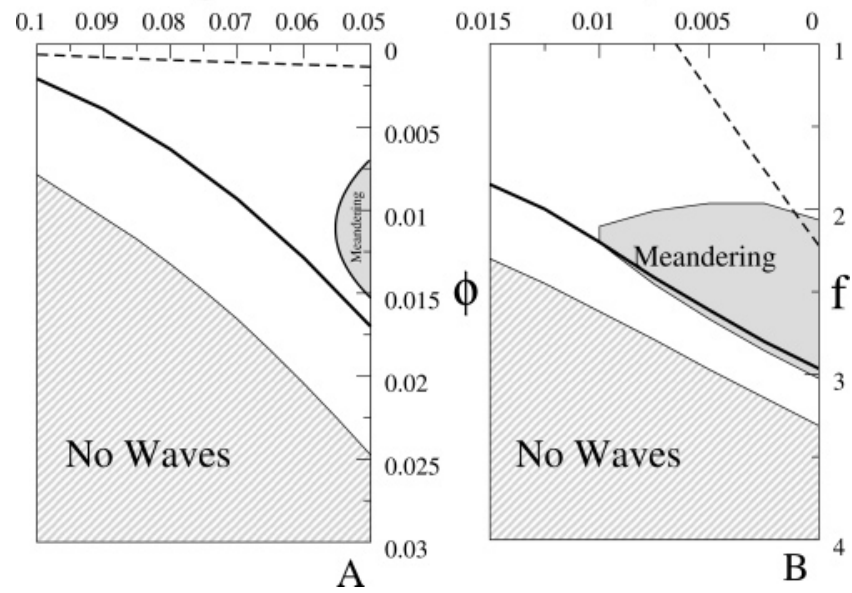

Figure 6. Phase diagrams of the Oregonator model in the parameter planes $(\phi, \epsilon)(\mathrm{A})$ and $(\phi, f)(\mathrm{B})$. The gray domain corresponds to meandering of spiral waves, the dashed light gray region to nonexcitable media, and the white area to rigidly rotating spiral waves. The medium is oscillatory above the dashed line and excitable below it. The bold line shows the boundary between the regions with positive and negative tension of the filament. The limits $\phi=0$ correspond to the dotted lines in Figure 2. The rest of parameters of the model are kept constant: $D_{u}$ $=1, D_{v}=0.6, q=0.002$ and (A) $f=2$, (B) $\epsilon=0.06$.

similar to that of Figure 6,A. However, the boundary between oscillatory and excitable behaviors depends on the illumination intensity $\phi$, while it was almost independent of the parameter $\epsilon$. The boundary between expansion and collapse of scroll rings coincides, as in the previous diagrams (Figure 2 and Figure 4), with the boundary between inward and outward loops of the corresponding 2D motion.

3.2. Three-Dimensional Simulations. The numerical simulations, used to determine the boundaries of the transition to negative tension in the phase diagrams, have been performed in the previous section assuming axial symmetry of scroll rings. Full 3D simulations are, however, necessary to investigate further evolution of the negative-tension instability and to observe the final turbulent state. An example of the evolution of a straight scroll wave under the conditions of the negativetension instability of its filament is shown in Figure 7. Here, the medium is weakly excitable, and spiral waves are rigidly rotating in the 2D medium with the same value of the parameters of the model. To perform this 3D simulation, a plane wave with a free cut edge is generated as the initial condition. The wave rolls around this linear defect and gives rise to the scroll wave rotating around a linear filament (Figure 7,A). To initiate the instability development, small perturbations must be applied. To generate them, weak external sinusoidal perturbation of the parameter $f$ along the scroll axis is introduced and maintained within a short time interval $12<t<12.75$. As a result of this perturbation, a small-amplitude modulation of the filament is created (Figure 7,B). Later on, the perturbation is removed, and the evolution starting with this perturbed state is monitored. After some initial relaxation (Figure 7,C,D), bumps gradually develop (Figure 7,E,F), and the filament acquires a complex shape (Figure 7,G,H). The filament breaks when it touches the boundaries of the system (Figure 7,I). Eventually, a set of short filaments is formed that snake out through the medium. The scroll waves rotate around these complex filaments and Winfree turbulence sets in Figure 7,J-L. The final state in Figure 7 is similar to the turbulent regimes previously observed for abstract reaction-diffusion models ${ }^{12-14}$ Although computer tomography methods allow for the reconstruction of 3D waves and the
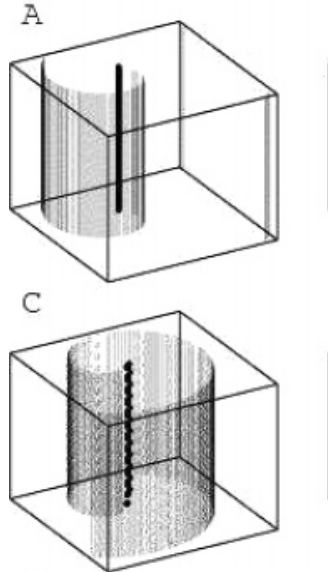

E

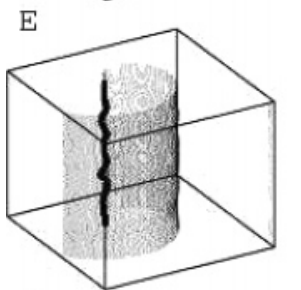

G

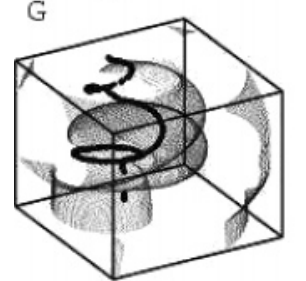

I

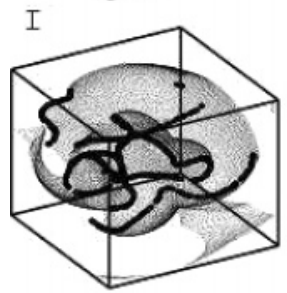

K

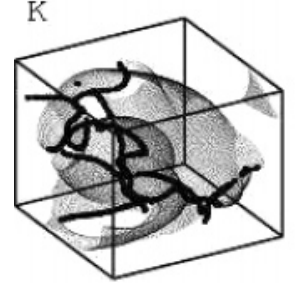

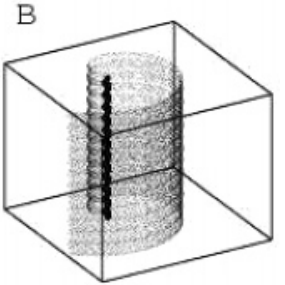

D

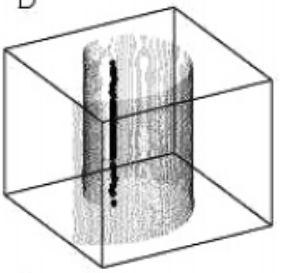

E

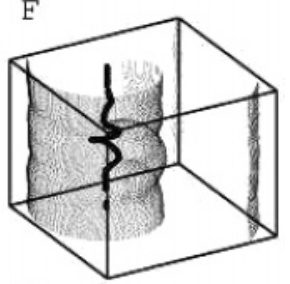

$\mathrm{H}$

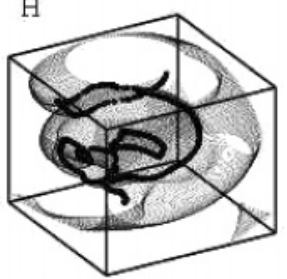

J

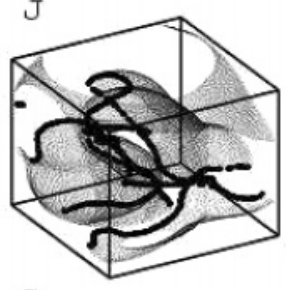

L

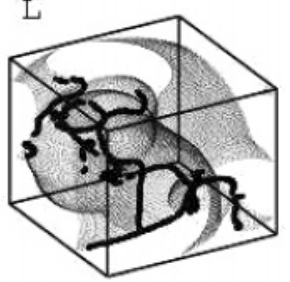

Figure 7. Evolution of a straight scroll wave and its filament under the negative-tension instability for weak excitability. To create the initial perturbation, small sinusoidal modulation of the parameter $f$ is introduced within a short interval $12<t<12.75$. The snapshots corresponds to (A) $t=10$, (B) $t=12.75$, (C) $t=15$, (D) $t=30$, (E) $t=45$, (F) $t=60$, (G) $t=75$, (H) $t=90$, (I) $t=105$, (J) $t=120$, (K) $t=135$, and (L) $t=150$. The parameters of the model are $f=2.80$, $\epsilon=0.07, D_{v}=0.6, \phi=0$, and $q=0.002$.

filaments from the experimental data are available, ${ }^{5,32}$ they have their own limitations, and, in the experiments, only planar projections of the actual 3D wave patterns are typically observed. ${ }^{23,33}$ In such experiments, the waves are viewed from the top of a thick layer of the medium with the BelousovZhabotinsky reaction. To facilitate comparison with such experimental data, we have also constructed in Figure 8 the planar projections of the 3D wave patterns shown in Figure 7. To obtain such projections, the reacting medium was assumed to be transparent. The local image density $U$ at a point $(x, y)$ in the planar projection was determined by the integration of the 

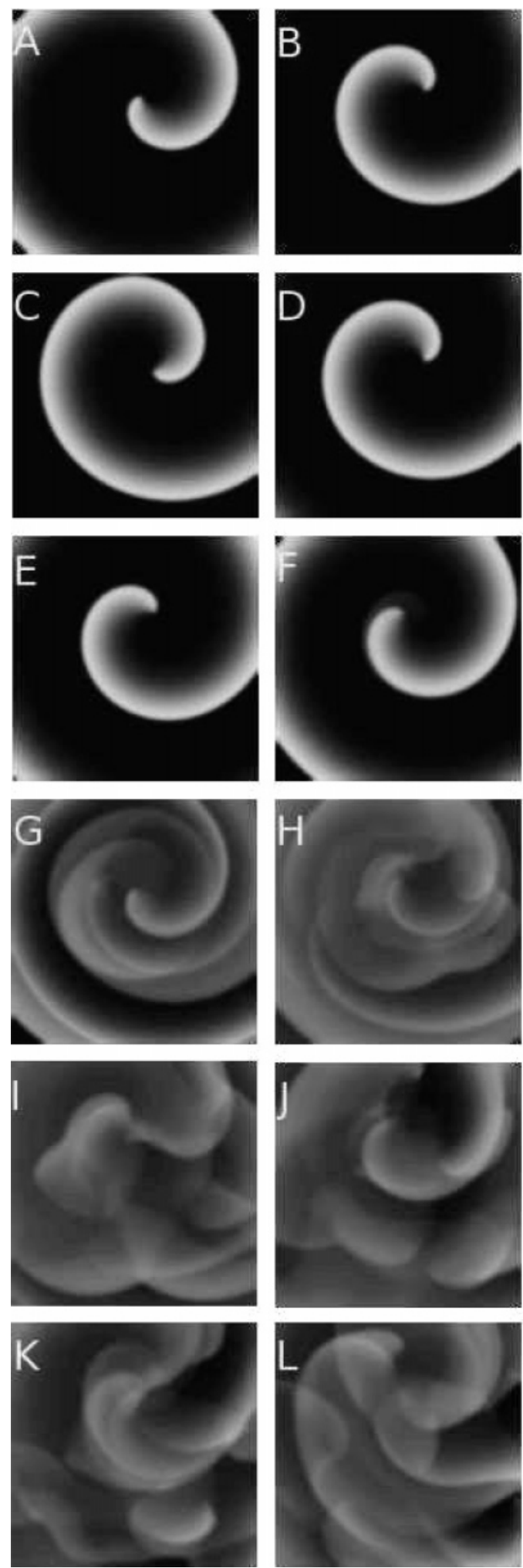

Figure 8. Evolution of a straight scroll wave under the negative-tension instability of its filament for weak excitability. The top view planar projection of the wave patterns is displayed (see the text); however, a nonlinear filter is applied to enhance the contrast. The model parameters and the time moments of the respective snapshots are the same as in Figure 7.

activator concentration $u$ along the vertical coordinate, $U(x, y)$ $=\int u(x, y, z) \mathrm{d} z$. White regions in the image correspond to higher local total concentrations $U$.

In the previous section, strong correlation between a transition from meandering with outward loops to meandering with inward
A

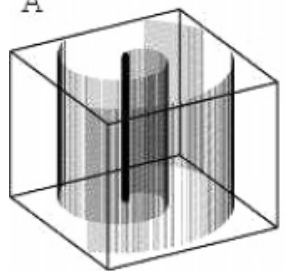

C

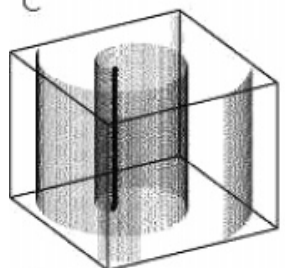

$\mathrm{E}$

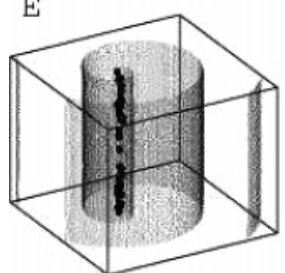

G

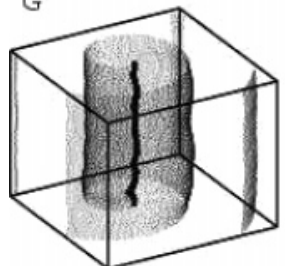

I

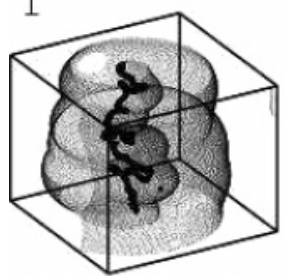

K

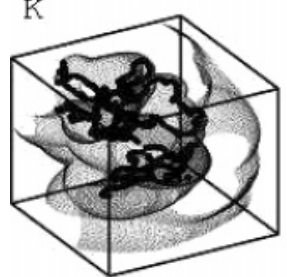

B

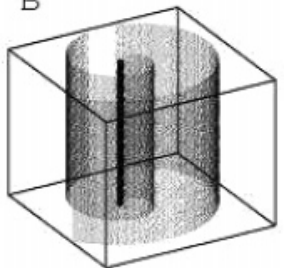

$\mathrm{D}$

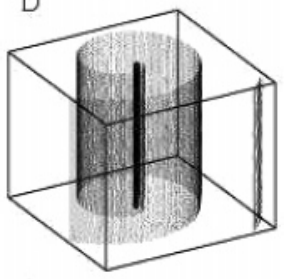

E

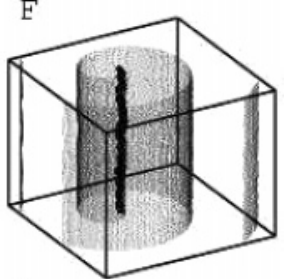

$\mathrm{H}$

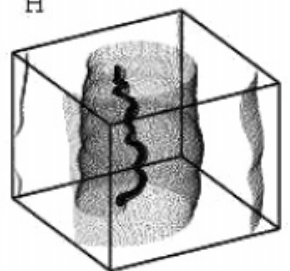

丁

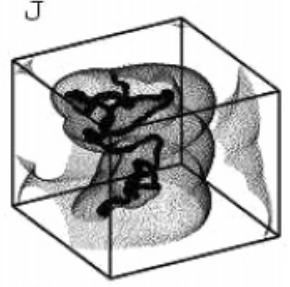

L

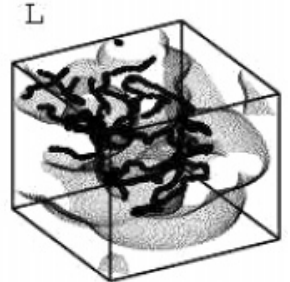

Figure 9. Evolution of a straight scroll wave and its filament under the meandering conditions for spiral waves in the respective $2 \mathrm{D}$ medium. To create the initial perturbation, small sinusoidal modulation of the parameter $f$ is introduced within a short interval $12<t<12.75$. The snapshots corresponds to (A) $t=10$, (B) $t=12.75$, (C) $t=15$, (D) $t=30$, (E) $t=75$, (F) $t=120$, (G) $t=165$, (H) $t=210$, (I) $t=$ 255 , (J) $t=300$, (K) $t=345$, and (L) $t=390$. The parameters of the model are $f=3.5, \epsilon=0.05, D_{v}=0, \phi=0$, and $q=0.002$.

loops for spiral waves in 2D medium and the negative-tension filaments in 3D was noticed. To further explore this relationship, 3D simulations of unstable scroll waves under meandering conditions have also been performed. The straight scroll wave, which initially rotates under meandering conditions with inward petals (Figure 9,A), is perturbed with a weak sinusoidal modulation in the parameter $f$ over a short time interval (Figure 9,B). After some initial relaxation of the perturbation (Figure 9,C), the filament begins to bend (Figure 9,D-I) and to develop a complex wave pattern (Figure 9,J-L). The density of filaments in the developing turbulent regime is larger than in Figure 7 
because the excitability is higher here. It should be noted that time scales are different in Figures 7 and 9, with the Winfree turbulence needing the longer time to develop under meandering conditions. Another important difference is that, similar to the situation with uniform periodic forcing of scroll waves (see ref 34), meandering leads to repeated self-crossing of the filament and budding of small scroll rings which grow and evolve. This kind of behavior was previously observed only in Winfree turbulence induced by slow periodic forcing. ${ }^{24}$ Simulations have also been performed under the condition of meandering with outward loops, when the negative-tension instability is absent. Initially, until the perturbation, moderate meandering is observed (Figure 10,A). After the application of the perturbation, the filament does not return to its straight shape, and some bumps persist during the entire evolution (Figure 10). Although these bumps move along the filament, they do not, however, grow with time, and the filament remains roughly straight. This behavior of filaments has been previously found for another model of excitable media (ref 35). If meandering is stronger, the effect becomes more pronounced, but the filament still remains connected and never spontaneously breaks if the boundaries are far away enough and the filament does not touch them (cf. ref 35).

3.3. Effects of Uniform Periodic Forcing. The photosensitive version of the Belousov-Zhabotinky reaction allows for the introduction of parametric forcing by periodic modulation of the illumination intensity. In the experiments, such forcing can never be spatially uniform, because of light attenuation in the medium. The actual spatial profile of the light intensity would depend on a particular experimental setup, and, in the present general study, we prefer not to take into account the effects of illumination gradients. Therefore, only the hypothetical uniform periodic modulation of light intensity will be considered here. This simplification would also allow us to perform comparison with the previous studies of uniform periodic forcing of Winfree turbulence in abstract models of excitable media. ${ }^{13,24}$ Under the conditions of weak excitability, scroll rings expand (Figure 11,A). When uniform periodic forcing is applied by modulation of the light intensity $\phi=\phi_{0}+\phi_{\mathrm{s}} \cos \left(2 \pi t / T_{\mathrm{f}}\right)$ with a period $T_{\mathrm{f}}$ shorter than the rotation period $T_{0}$ of the waves around their filament $\left(T_{\mathrm{f}}<T_{0}\right)$ and high enough amplitude $\phi_{\mathrm{s}}$, the expansion is changed into the collapse of the scroll ring (Figure 11,A), in agreement with previous results. ${ }^{13}$ While the unforced system presents only one frequency, the periodically forced scroll ring develops a complex motion composed of two frequencies, as can be observed in the Fourier spectrum (Figure $11, \mathrm{~B})$. The new frequency is the difference between the forcing frequency and the frequency of rotation around the filament $\omega_{\mathrm{f}}$ $-\omega_{0}^{\prime}$. It is also important to note that forcing shifts the main rotation frequency, as seen in Figure 11,B. While the unforced scroll ring rotates around the filament with $T_{0}=8.2$, the forced scroll ring rotates with $T_{0}^{\prime}=9.6 .^{24}$ These numerical simulations have been performed in the reduced 2D geometry, assuming axial symmetry of the filament, and they correctly describe only the initial stage of the instability development.

Full 3D simulations of the wave patterns under periodic uniform modulation of the illumination intensity have also been performed. When forcing was applied from the beginning of the simulation, the filament did not undergo an evolution to chaotic dynamics and remained roughly straight, with some helicoidal modulation. If forcing is applied after the filament has already developed a significant shape variation (Figure $12, \mathrm{~A}-\mathrm{D})$, it is able to prevent further development of the instability and make its shape more regular (Figure 12, $\mathrm{E}^{\prime}-\mathrm{L}^{\prime}$ ).
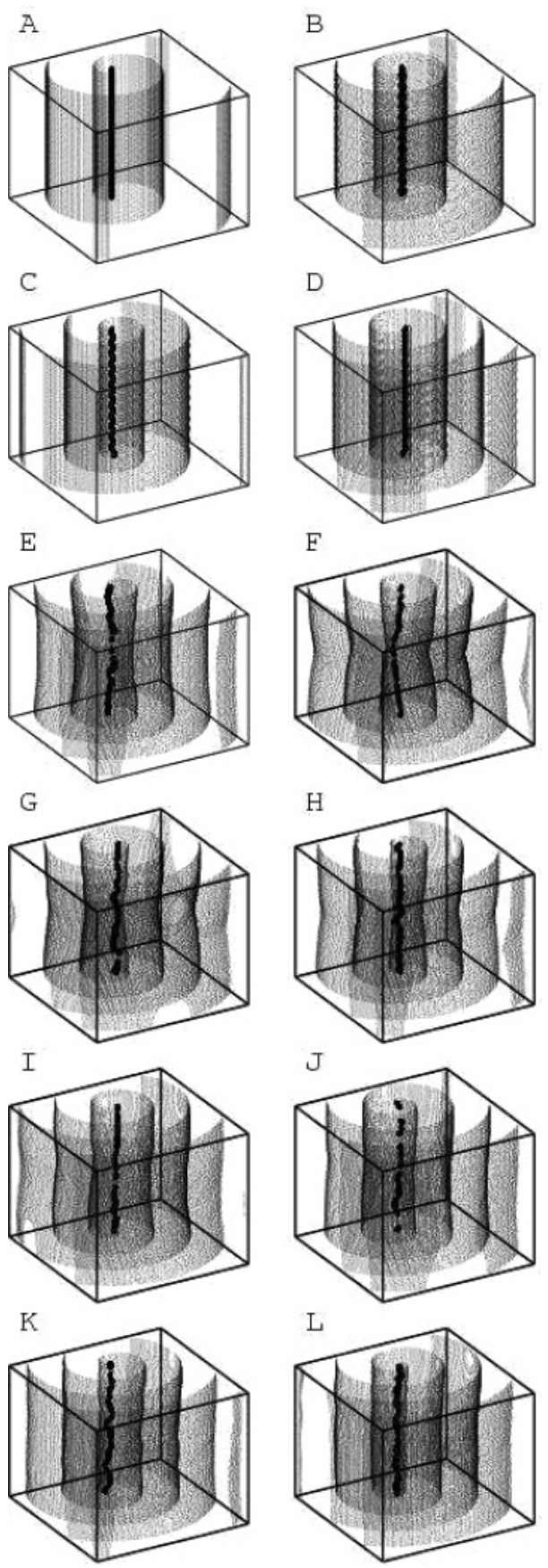

L

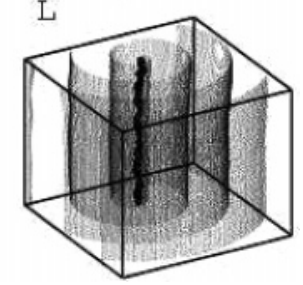

Figure 10. Evolution of a straight scroll wave and its filament under the meandering conditions in the absence of the negative-tension instability. To create the initial perturbation, small sinusoidal modulation of the parameter $f$ is introduced within a short interval $12<t<12.75$. The snapshots corresponds to (A) $t=10$, (B) $t=12.75$, (C) $t=15$, (D) $t=30$, (E) $t=75$, (F) $t=120$, (G) $t=165$, (H) $t=210$, (I) $t=$ 255 , (J) $t=300$, (K) $t=345$, and (L) $t=390$. The parameters of the model are $f=2.5, \epsilon=0.05, D_{v}=0, \phi=0$, and $q=0.002$.

Under the same conditions without forcing, transition to turbulent dynamics is observed (Figure 12,E-H). Note that, under forcing, the parameter $\phi$ varies inside the interval $\phi_{0} \pm$ $\phi_{\mathrm{s}}$, always remaining within the region in the phase diagram in Figure 6 where negative tension is observed.

Within the computation time in Figure 12, the periodic forcing does not return the filament to the initial straight configuration. This may be related to the fact that, as seen in Figure $6, \mathrm{~B}$, forcing increases the main rotation period from $T_{0}$ to $T_{0}^{\prime}$, and, the system moves away from the resonance making the forcing less efficient (see the analysis in ref 24). Employing forcing 

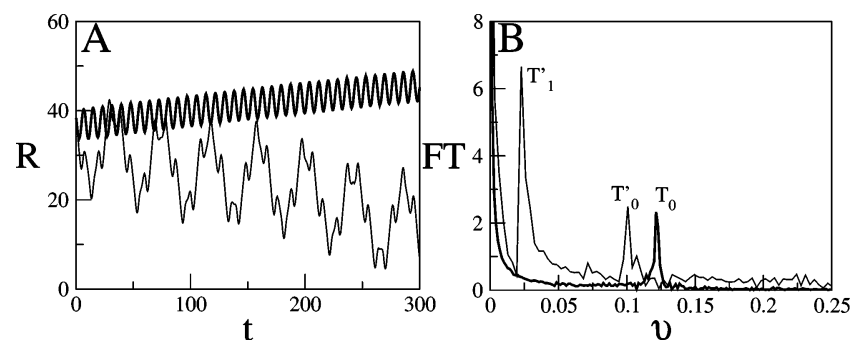

Figure 11. Effects of uniform rapid periodic forcing of expanding scroll rings. (A) Dependence of the radius $R$ of a scroll ring on time without forcing (thick line) and under forcing with $\phi_{\mathrm{s}}=0.0025$ and $T_{\mathrm{f}}$ $=8$ (thin line). (B) Power spectra of the temporal signals in panel A. Without forcing (thick line), the single power peak is located at $T_{0}=$ 8.2. When forcing is applied (thin line), the peaks appear at $T_{0}^{\prime}=9.6$ and $T_{1}^{\prime}=56.2$. The model parameters are $f=2.0, \epsilon=0.065, D_{v}=$ $0.6, \phi_{0}=0.0150$, and $q=0.002$.

with a larger period $T_{\mathrm{f}}\left(T_{0}<T_{\mathrm{f}}<T_{0}^{\prime}\right)$, which is closer to the actual rotation period $T_{0}^{\prime}$, we could see that such forcing leads the scroll wave to eventually rotate around a straight filament.

\section{Discussion and Conclusions}

It would be very interesting to find the intricate and impressive 3D patterns of Winfree turbulence in the BelousovZhabotinsky reaction. Until recently, these kinds of patterns have been seen only in numerical simulations of abstract models of excitable reaction-diffusion systems. In our study, we have performed numerical simulations of the realistic two-variables
Oregonator model of the Belousov-Zhabotinsky reaction and demonstrated that there are regions in the parameter space of this model where scroll filaments are unstable due to the negative tension. These regions are determined in terms of the parameters $f, \phi$, and $\epsilon$. Although there is not direct correspondence between these parameters of the Oregonator model and chemical concentrations, qualitative relations between them are known. ${ }^{26}$ Therefore, we hope that our study would help to design experiments in the Belousov-Zhabotinsky reaction aimed at finding the negative-tension instability of filaments and Winfree turbulence in this classical system.

The phase diagram is divided into domains with excitable and oscillatory dynamics. It should be noted that the properties of propagation of spiral and scroll waves in the oscillatory parameter region do not change much with respect to those in the excitable domain. At large values of $\epsilon$, the boundaries of the negative-tension instability and of the transition to oscillatory dynamics are crossing (see Figures 2 and 4). Therefore, unstable filaments may also be observed in the oscillatory version of the Belousov-Zhabotinsky reaction. Filament instabilities in the oscillatory systems described by the complex GinzburgLandau equation have previously been considered. ${ }^{36}$

The dynamics of scroll waves under meandering conditions is not completely understood, and various kinds of complex behavior have been observed in previous numerical studies. ${ }^{35}$ Our numerical investigations indicate a clear relation between the condition of straight drift of spiral waves, which separates meandering spirals with inward and outward loops, and the

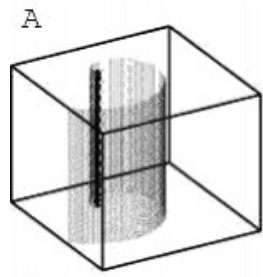

E

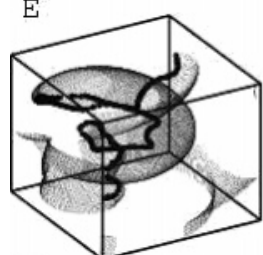

$\mathrm{E}^{\prime}$

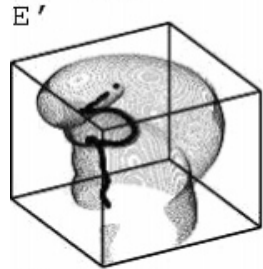

$I^{\prime}$

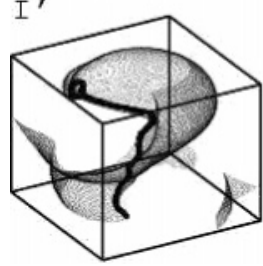

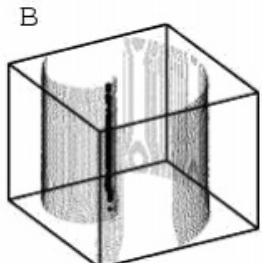

E

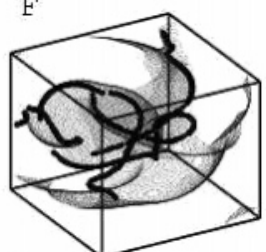

$\mathrm{E}^{\prime}$

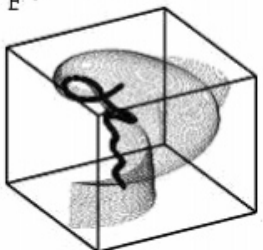

$J^{\prime}$

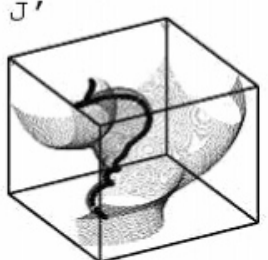

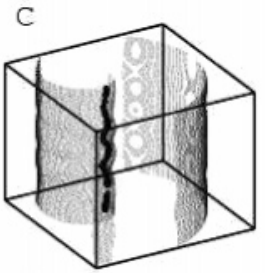

G

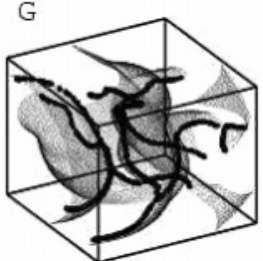

$\mathrm{G}^{\prime}$

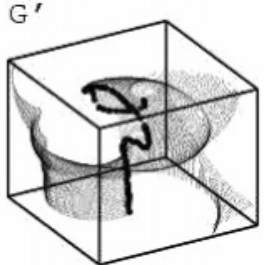

$\mathrm{K}^{\prime}$

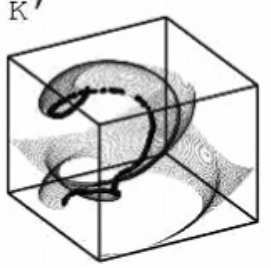

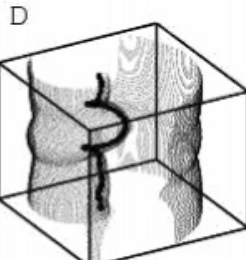

$\mathrm{H}$

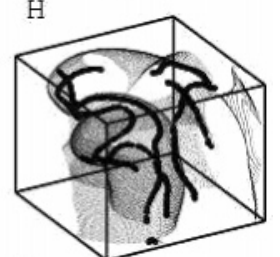

$H_{r}$
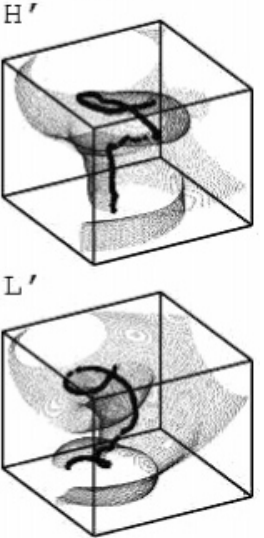

Figure 12. Evolution of a straight scroll wave and its filament under conditions of negative tension instability, without (A-H) and with uniform periodic forcing $\left(\mathrm{E}^{\prime}-\mathrm{L}^{\prime}\right)$. To create the initial perturbation, small sinusoidal modulation of the parameter $f$ is introduced within a short interval 12 $<t<12.75$. Snapshots (A-D) show the instability development. At time $t=90$ (snapshots D), uniform periodic forcing in the parameter $\phi$ is applied, and the evolution of the wave patterns is monitored (snapshots $\mathrm{E}^{\prime}-\mathrm{L}^{\prime}$ ). For comparison, snapshots $\mathrm{E}-\mathrm{H}$ show the continued behavior of the wave pattern if the forcing has not been applied. The shown snapshots corresponds to the time moments (A) $t=15$, (B) $t=30$, (C) $t=60$, (D) $t=90,\left(\mathrm{E}, \mathrm{E}^{\prime}\right) t=120,\left(\mathrm{~F}, \mathrm{~F}^{\prime}\right) t=150,\left(\mathrm{G}, \mathrm{G}^{\prime}\right) t=180,\left(\mathrm{H}, \mathrm{H}^{\prime}\right) t=210,\left(\mathrm{I}^{\prime}\right) t=270,\left(\mathrm{~J}^{\prime}\right) t=330,\left(\mathrm{~K}^{\prime}\right) t=390$, and $\left(\mathrm{L}^{\prime}\right) t=450$. The parameters of the model are $f=2.0, \epsilon=0.065, D_{v}=0.6, \phi_{0}=0.0150$, and $q=0.002$. 
condition of zero tension, which separates regions with expansion and collapse of scroll rings. A similar relationship has been also obtained by us in the Barkley model, and it was sketched in ref 9 for a reduced model of cardiac tissue. While the direction of the loops in the meandering motion of spirals is a $2 \mathrm{D}$ effect, the negative-tension instability of the filaments is a purely 3D property. Remarkably, both effects are nonetheless related. In a previous study, ${ }^{37}$ the condition of negative tension for rigidly rotating scrolls was associated with a resonance between the meandering and translation modes. However, this study was done outside of the meandering domain, and the dynamics inside the region of meandering was not discussed there.

This empirical relation can be helpful in the experiments aimed at finding the negative-tension instability. Although meandering with outward loops has been extensively studied in the Belousov-Zhabotinsky reaction, ${ }^{28,38,39}$ there are only a few experimental observations of the other type of meandering. It was clearly seen in the photosensitive version of the reaction ${ }^{40}$ and in the standard Belousov-Zhabotinsky reaction in porous media. ${ }^{41}$ Typically, diffusion coefficients of the activator and the inhibitor species in the experiments with aqueous solutions are not much different, and, as we have shown, the region with inward meandering should be reduced in size in this case. In ref 40 , the reaction was taking place in a silicahydrogel gel which reduced the diffusion of the inhibitor. In ref 41 , the reaction taking place in a porous media has also probably a reduced inhibitor diffusion.

We would like to note additionally that the relation between the type of meandering and the filament tension of scroll waves can be important for the understanding of wave instabilities in the cardiac tissue. In the cardiac tissue, waves usually undergo strong meandering with several involved frequencies. Here this relation could guide in order to find regions of negative tension at least in numerical models of cardiac tissue because each frequency involved in the complex motion in $2 \mathrm{D}$ could give rise to different contributions in the 3D dynamics.

An important property of the Belousov-Zhabotinsky reaction is that, in its photosensitive version, this reaction is amenable to control by varying the illumination intensity which is directly related to the parameter $\phi$ of the Oregonator model. We have shown that the forcing of scroll waves by periodic uniform modulation of the illumination intensity can be used to control the negative-tension instability and suppress Winfree turbulence of scroll waves in the photosensitive Belousov-Zhabotinsky reaction, similar to what has previously been shown in refs 13 and 24 for the abstract Barkley model. In the experiments, a gradient of the illumination intensity inside the volume is always present. ${ }^{23} \mathrm{We}$ expect however that, at least for relatively small gradients, the effects of periodic forcing would be qualitatively the same as in the ideal case of uniform forcing, which has been considered here. We would like to note that forcing with periodic variation of an applied electric field can also be employed to control 3D wave patterns.

Acknowledgment. Stimulating discussions with Q. Ouyang, K. Showalter, C. Luengviriya, and M. Hauser are gratefully acknowledged. One of us (S.A.) acknowledges the EU financial support in the framework of the EU Marie Curie Research and Training Network "Universal Principles of Pattern Formation".

\section{References and Notes}

(1) Zaikin, A. N.; Zhabotinsky, A. M. Nature 1970, 225, 535.

(2) Winfree, A. T. Science 1972, 175, 634.

(3) Ouyang, Q.; Flesselles, J. M. Nature (London) 1996, 379, 143.

(4) Winfree, A. T. Science 1973, 181, 937.

(5) Storb, U.; Neto, C. R.; Bär, M.; Müller, S. C. Phys. Chem. Chem. Phys. 2003, 5, 2344. 7508.

(7) Tyson, J. J.; Fife, P. C. J. Chem. Phys. 1980, 73, 2224.

(8) Davidenko, J. M.; Pertsov, A. V.; Salomonsz, R.; Baxter, W.; Jalife, J. Nature (London) 1992, 355, 349.

(9) Fenton, F. H.; Cherry, E. M.; Hastings, H. M.; Evans, S. J. Chaos 2002, $12,852$.

(10) Winfree, A. T. Science 1994, 266, 1003.

(11) Panfilov, A. V.; Rudenko, A. N. Physica D (Amsterdam) 1987, $28,215$.

(12) Biktashev, V. N.; Holden, A. V.; Zhang, H. Philos. Trans. R. Soc. London, Ser. A 1994, 347, 611.

(13) Alonso, S.; Sagués, F.; Mikhailov, A. S. Science 2003, 299, 1722.

(14) Alonso, S.; Kähler, R.; Mikhailov, A. S.; Sagués, F. Phys. Rev. E 2004, 70, 056201

(15) Field, R. J.; Noyes, R. M.; Koros, E. J. Am. Chem. Soc. 1972, 94, 8649.

(16) Agladze, K. I.; Kocharyan, R. A.; Krinky, V. I. Physica D (Amsterdam) 1991, 49, 1 .

(17) Braznhik, P. K.; Davydov, V. A.; Zykov, V. S.; Mikhailov, A. S. Sov. Phys. JETP 1987, 66, 984.

(18) Zaritski, R. M.; Mironov, S. F.; Pertsov, A. M. Phys. Rev. Lett.

2004, 92,168302

(19) Mikhailov, A. S.; Showalter, K. Phys. Rep. 2006, 425, 79.

(20) Steinbock, O.; Zykov, V.; Müller, S. C. Nature (London) 1993 $366,322$.

(21) Sakurai, T.; Mihaliuk, E.; Chirila, F.; Showalter, K. Science 2002, 296, 2009.

(22) Vinson, M.; Mironov, S.; Mulvey, S.; Pertsov, A. Nature 1997, $386,477$.

(23) Amemiya, T.; Kettunen, P.; Kádár, S.; Yamaguchi, T.; Showalter, K. Chaos 1999, 8, 872 .

(24) Alonso, S.; Sagués, F.; Mikhailov, A. S. Chaos 2006, 16, 023124. (25) Krug, H. J.; Pohlmann, L.; Kuhnert, K. J. Phys. Chem. 1990, 94 4862.

(26) Jahnke, W.; Winfree, A. T. Int. J. Bif. Chaos 1991, 1, 445.

(27) Yamaguchi, T.; Kuhnert, L.; Nagy-Ungvarai, Zs.; Müller, S. C.; Hess, B. J. Phys. Chem. 1991, 95, 5831.

(28) Jahnke, W.; Skaggs, W. E.; Winfree, A. T. J. Phys. Chem. 1989 93,740 .

(29) Fenton F.; Karma, A. Phys. Rev. Lett. 1998, 81, 481.

(30) Broens, M.; Bar-Eli, K. J. Phys. Chem. 1991, 95, 8706.

(31) Panfilov, A. V.; Rudenko, A. N.; Krinsky, V. I. Biophysics 1986, 31,850 .

(32) Pertsov, A.; Vinson, M.; Muller, S. C. Physica D (Amsterdam) 1993 $63,233$.

(33) Zhang, C. X.; Zhang, H.; Ouyang, Q.; Hu, B. B.; Gunaratne, G. H. Phys. Rev. E 2003, 68, 036202.

(34) Fiedler, B.; Mantel, R. M. Doc. Math 2000, 5, 695.

(35) Rusakov, A.; Medvinsky, A. B.; Panfilov, A. V. Phys. Rev. E 2005 , 72,022902 .

(36) Aranson, I. S.; Bishop, A. R.; Kramer, L. Phys. Rev. E 1998, 57, 5276.

(37) Henry, H. Phys. Rev. E 2004, 70, 026204.

(38) Skinner, G. S.; Swinney, H. L. Physica D (Amsterdam) 1991, 48,

(39) Plesser, T.; Müller S. C.; Hess, B. J. Phys. Chem. 1990, 94, 7501.

(40) Braune, M.; Engel, H. Chem. Phys. Lett. 1993, 204, 257.

(41) Li, G.; Ouyang, Q.; Petrov, V.; Swinney, H. Phys. Rev. Lett. 1996, $77,2105$. 